\title{
Contraception for the HIV-Positive Woman: A Review of Interactions between Hormonal Contraception and Antiretroviral Therapy
}

\author{
Jennifer A. Robinson, Roxanne Jamshidi, and Anne E. Burke \\ Department of Gynecology and Obstetrics, Johns Hopkins Bayview Medical Center, 4940 Eastern Avenue, \\ A Building, Room 121, Baltimore, MD 21224, USA \\ Correspondence should be addressed to Jennifer A. Robinson, jrobin87@jhmi.edu
}

Received 21 February 2012; Accepted 15 May 2012

Academic Editor: Deborah Cohan

Copyright ( $\odot 2012$ Jennifer A. Robinson et al. This is an open access article distributed under the Creative Commons Attribution License, which permits unrestricted use, distribution, and reproduction in any medium, provided the original work is properly cited.

\begin{abstract}
Background. Preventing unintended pregnancy in HIV-positive women can significantly reduce maternal-to-child HIV transmission as well as improve the woman's overall health. Hormonal contraceptives are safe and effective means to avoid unintended pregnancy, but there is concern that coadministration of antiretroviral drugs may alter contraceptive efficacy. Materials and Methods. We performed a literature search of PubMed and Ovid databases of articles published between January 1980 and February 2012 to identify English-language reports of drug-drug interactions between hormonal contraceptives (HCs) and antiretroviral drugs (ARVs). We also reviewed the FDA prescribing information of contraceptive hormone preparations and antiretrovirals for additional data and recommendations. Results. Twenty peer-reviewed publications and 42 pharmaceutical package labels were reviewed. Several studies of combined oral contraceptive pills (COCs) identified decreased serum estrogen and progestin levels when coadministered with certain ARVs. The contraceptive efficacy of injectable depot medroxyprogesterone acetate (DMPA) and the levonorgestrel intrauterine system (LNG-IUS) were largely unaffected by ARVs, while data on the contraceptive patch, ring, and implant were lacking. Conclusions. HIV-positive women should be offered a full range of hormonal contraceptive options, with conscientious counseling about possible reduced efficacy of COCs and the contraceptive implant when taken with ARVs. DMPA and the LNG-IUS maintain their contraceptive efficacy when taken with ARVs.
\end{abstract}

\section{Introduction}

The face of the HIV/AIDS epidemic has changed dramatically since its emergence in the 1980s. Far from its origins as an illness of homosexual men, HIV/AIDS is increasingly affecting women around the world: in 2008, women made up nearly half of the global population of those infected with HIV (15.7 million women, 33.4 million total) [1]. While spread of the epidemic has slowed, addressing the health needs of women infected with HIV remains an important priority. Recent efforts have largely focused on expanding access to HIV diagnosis and counseling, as well as treatment with highly-active antiretroviral therapy (HAART). Providing reproductive health services to women living with HIV is crucial to improving their overall health. Preventing unplanned or mistimed pregnancy allows a woman with HIV to optimize her own health and has the potential to decrease maternal-to-child transmission of HIV. The World Health Organization (WHO) reports that approximately $90 \%$ of children living with HIV acquired the infection perinatally_during pregnancy, birth, or breastfeeding [1]. In a model comparing interventions to decrease maternalto-child transmission (MTCT) of HIV, increasing use of contraception was found to prevent $28.6 \%$ more HIVpositive births than increasing use of peripartum nevirapine [2]. Effective contraception thus offers great opportunity to slow the spread of perinatally acquired HIV, though sexual transmission of HIV may still occur between serodiscordant couples. 
As access to both modern methods of contraception and antiretroviral drugs (ARVs) expands, women with HIV enter the largely uncharted territory of potential drug interactions. In this paper we will summarize the available literature regarding coadministration of ARVs and hormonal contraception, with a focus on whether ARVs lead to alterations in hormonal contraceptive efficacy.

Modern hormonal birth control methods available in the United States include daily pills (combined oral contraceptives (COCs) that contain estrogen and a progestin, as well as progestin-only pills (POPs)), a weekly combined hormonal patch, a monthly combined hormonal vaginal ring, injectable depot medroxyprogesterone acetate (DMPA) given every three months, a three-year etonogestrel (progestin) implantable rod, and a five-year levonorgestrel intrauterine system (LNG-IUS). The latter two are often described as long-acting reversible contraceptives (LARCs). All of these methods are highly effective at preventing pregnancy, with the typical-use failure rate ranging from $0.1 \%$ (LNG-IUS) to $8 \%$ (POPs, COCs, ring) [3]. Hormonal emergency contraceptives (ECs) are also available, which reduce the risk of pregnancy by $89 \%$ when taken within 72 hours of unprotected sex [3]. Contraceptive hormones are metabolized by the hepatic cytochrome (CYP) P450 pathway, which is also responsible for the metabolism of many ARVs [4]. Orally administered contraceptive hormones are subject to extensive first-pass gut and hepatic metabolism, which necessitates higher estrogen and progestin doses than those required when these hormones are given via routes that lead to minimal first-pass metabolism [5]. Whether novel delivery systems (i.e., transdermal or transvaginal) affect the pharmacokinetics of contraceptive hormones, and how these drugs will interact with ARVs, remains unclear. It is also uncertain how LARC methods interact with HAART regimens.

Contraceptive steroids mainly work by negative feedback inhibition of the hypothalamic-pituitary-ovarian (HPO) axis, with additional effects on cervical mucus and the endometrium. Exogenous estrogen and progestin both profoundly suppress pulsatile secretion of follicle-stimulating hormone (FSH) and luteinizing hormone ( $\mathrm{LH})[6,7]$. However, most of the contraceptive effects (i.e., ovulation suppression, thinning of the endometrium, and cervical mucus thickening) are due primarily to progestin and are dose-dependent $[6,8]$. Ethinyl estradiol (EE), the estrogenic component of most currently marketed COCs, is primarily metabolized through the hepatic CYP pathway. Specifically, hydroxylation of EE is catalyzed by the hepatic enzymes CYP3A4 and CYP2C9. Wide variation in the levels of these enzymes among individuals is thought to contribute significantly to large intersubject variability of EE pharmacokinetics [9]. The contraceptive progestins available today also vary widely in their metabolism and pharmacokinetics, with large intersubject and intrasubject variability [10].

Modern management of HIV typically involves a combination of ARVs, commonly described as highly active antiretroviral therapy (HAART), since monotherapy can lead to the development of viral resistance to drug therapy. In treatment-naive patients, a starting regimen typically includes two nucleoside reverse transcriptase inhibitors (NRTIs) and a protease inhibitor (PI), two NRTIs and a nonnucleoside reverse transcriptase inhibitor (NNRTI), or three NRTIs [11]. Table 1 lists the five preferred regimens described by the Department of Health and Human Services (DHHS). Ritonavir, a protease inhibitor, is rarely used for its own antiretroviral activity, but is used for its inhibitory effect on CYP3A4, the liver enzyme that normally metabolizes protease inhibitors. A low dose of ritonavir is used to enhance, or "boost," other protease inhibitors, thereby improving ARV efficacy.

This paper will examine the available evidence regarding drug interactions between ARVs and all types of hormonal contraception (HC), including transdermal and transvaginal routes as well as LARC options. Pertinent pharmacokinetic (PK) parameters, such as the maximum serum concentration $\left(C_{\max }\right)$, minimum serum concentration $\left(C_{\min }\right)$, half-life $\left(t_{1 / 2}\right)$, and the area under the concentration-time curve (AUC), will be discussed for each drug interaction, when available. We will also include recommendations made in the Centers for Disease Control and Prevention Medical Eligibility Criteria (CDC MEC). This tool provides evidence-based recommendations about appropriate use of contraceptives in women with various medical comorbidities, including HIV. The document cross-references contraceptive methods and medical conditions, assigning a recommendation category to each combination of these. These categories are as follows: Category $1-$ a condition for which there is no restriction for use of the contraceptive method; Category 2-a condition for which the advantages of using the method generally outweigh the theoretical or proven risks; Category 3-a condition for which the theoretical or proven risks usually outweigh the advantages of using the method; Category 4a condition that represents an unacceptable health risk if the contraceptive method is used [35].

\section{Materials and Methods}

We performed a literature search using PubMed and Ovid databases to identify English-language articles published from January 1980 to February 2012. Search terms included contraception, hormonal contraception, contraceptives, hormonal contraceptives, birth control, HIV, family planning, and the generic names of individual ARVs and HC components: abacavir, amprenavir, atazanavir, darunavir, delavirdine, didanosine, efavirenz, emtricitabine, enfuvirtide, etravirine, fosamprenavir, indinavir, lamivudine, lopinavir, maraviroc, nelfinavir, nevirapine, raltegravir, rilpivirine, ritonavir, saquinavir, stavudine, tenofovir, tipranavir, zalcitabine, zidovudine, ethinyl estradiol, ethynodiol diacetate, etonogestrel, levonorgestrel, depot medroxyprogesterone acetate, norethindrone, desogestrel, drospirenone, norgestrel, norgestimate, mifepristone, and ulipristal. We included randomized and nonrandomized trials, observational studies, and case reports that provided pharmacokinetic data for any of the hormonal contraceptives. Hand searches of relevant journals, references 
TABLE 1: DHHS preferred antiretroviral regimens for antiretroviral therapy-naïve patients [11].

NNRTI-based regimen

PI-based regimens

INSTI-based regimen

Preferred regimen for pregnant women
Efavirenz (NNRTI) + tenofovir (NRTI) + emtricitabine (NRTI)

Atazanavir/ritonavir (PI) + tenofovir (NRTI) + emtricitabine (NRTI)

Darunavir/ritonavir (once daily) (PI) + tenofovir (NRTI) + emtricitabine (NRTI)

Raltegravir (II) + tenofovir (NRTI) + emtricitabine (NRTI)

Lopinavir/ritonavir (twice daily) (PI) + zidovudine (NRTI) + lamivudine (NRTI)

Abbreviations. NNRTI: nonnucleoside reverse transcriptase inhibitor; NRTI: nucleos(t)ide reverse transcriptase inhibitor; PI: protease inhibitor; INSTI (II): integrase strand transfer inhibitor.

from review articles, and conference abstracts were performed, and pharmaceutical package labels for individual drugs/methods were also reviewed. Results are presented according to type of contraceptive method.

\section{Results}

A total of 20 published studies or abstracts were identified and included, as were 42 FDA-mandated package labels published by pharmaceutical companies. Package labels that did not include any data about drug interactions between hormonal contraceptives and ARVs are not reported.

\subsection{Combined (Estrogen + Progestin) Methods}

3.1.1. Combined Oral Contraceptives (COCs). A review of the FDA labeling of a selection of COC yields mixed advice about interactions with antiretroviral drugs. Labels for three COCs (EE/norgestrel (Lo/Ovral), EE/levonorgestrel (Alesse), and EE/norgestimate (Ortho Tri-Cyclen)) mention possible interaction with protease inhibitors (PI) and refer providers to the PI label for additional information [36-38]. Labels for two other COCs (EE/drospirenone (Yaz) and EE/desogestrel (Mircette)) warn of potential decreased contraceptive efficacy when taken with enzyme-inducing drugs such as anticonvulsants or rifampin, but there is no reference to specific studies about potential interactions with ARV $[39,40]$.

The package labels of various ARVs offer some more detailed information. Of the ten PIs available in the United States, all package labels provide data regarding interactions between the PI and COCs. Seven PIs (amprenavir, darunavir, lopinavir, nelfinavir, tipranavir, saquinavir, and ritonavir) were associated with decreases in pharmacokinetic parameters of both estrogen and progestin components of the coadministered COC, and the labels consequently recommended that women use an additional barrier method or rely on nonhormonal contraceptives [13, 14, 41-45]. These conclusions emphasized the decrease in serum EE concentrations as a reason to avoid COCs, despite the fact that most contraceptive activity of COCs is provided by the progestin.

Atazanavir has differing effects on COC metabolism, depending on whether or not it is coadministrated with ritonavir. When women took EE/norethindrone (NET) and atazanavir alone on days 16 to 29 of the COC cycle, there was an increase in the serum concentrations of both
EE and norethindrone (Table 2) [12]. However, when atazanavir combined with ritonavir was coadministrated with EE/norgestimate on days 29 to 42 after a full cycle of the COC, concentrations of EE were decreased while levels of norgestimate were increased. The manufacturer of atazanavir advises that women use a pill with at least $35 \mathrm{mcg}$ $\mathrm{EE}$ in the setting of ritonavir-boosted atazanavir, but to use a pill with no more than $30 \mathrm{mcg}$ of $\mathrm{EE}$ if atazanavir was the sole protease inhibitor [12]. These recommendations are potentially confusing for practitioners seeking clinical advice.

The earliest peer-reviewed study identified in our paper was a 1998 open-label trial of ritonavir and a COC containing EE/ethynodiol diacetate [16]. A total of 23 healthy, HIV-negative women were included in the analysis, each of whom received a dose of the COC on study days 1 and 29. From day 16 to 29 , subjects took escalating doses of ritonavir to a maximum dose of $500 \mathrm{mg}$ every 12 hours. Serial blood samples were collected over 48 hours following each dose of COC. Compared to the COC dose given on study day 1 , the dose given on day 29 resulted in a 32\% lower EE mean $C_{\max }$ $(P<0.001)$ and $41 \%$ lower mean AUC $(P<0.001)$. The authors noted that one subject who missed the morning dose of ritonavir on day 29 still showed a 31\% decrease in EE AUC, "suggesting enzyme induction rather than altered absorption as the probable cause of the interaction" [16].

Mildvan et al. studied the interactions between steadystate nevirapine and a single dose of EE/NET [18]. Fourteen HIV-positive women aged 26 to 47 years with a stable ARV regimen for at least 4 weeks were enrolled, and 10 completed the study. Subjects received one dose of the study COC on day 0 , followed by intense plasma sampling over the next 48 hours. They then received nevirapine for the next 28 days, with typical escalation of the dose from week 2 to week 3 (Table 2). They received a second dose of the COC on day 30 along with the scheduled dose of nevirapine, followed by additional plasma sampling. Participants continued ARV therapy during the study period. Compared to the COC taken alone, EE administered with nevirapine had a $29 \%$ decrease in median AUC $(P=0.014)$, but no change in $C_{\max }$, and a decrease in mean terminal half-life from 16.6 hours to 12.5 hours $(P=0.010)$. Norethindrone demonstrated an $18 \%$ decrease in median AUC when COC was given with nevirapine, compared to the COC given alone $(P=0.016)$. The authors concluded that nevirapine increases the systemic clearance of EE and NET, with the potential of lowering concentrations to subtherapeutic levels. The authors provide no definition of what constitutes a subtherapeutic level of EE or norethindrone. They suggest that, in the absence of 
TABLE 2: Summary of pharmacokinetic interactions (results are given as geometric mean ratios of HC + ARV to HC alone, with $90 \%$ confidence intervals, unless otherwise specified).

\begin{tabular}{|c|c|c|}
\hline $\begin{array}{l}\text { Drug } \\
\text { Subclass } \\
\end{array}$ & $\begin{array}{c}\text { Source and number of } \\
\text { patients }\end{array}$ & Effect on hormonal contraceptives \\
\hline \multirow[t]{15}{*}{$\begin{array}{l}\text { Atazanavir (Reyataz) } \\
\text { Protease inhibitor }\end{array}$} & $\begin{array}{l}\text { Reyataz package label } \\
{[12]}\end{array}$ & \\
\hline & & $\begin{array}{l}\text { EE } 35 \mathrm{mcg} / \mathrm{NET}(0.5 \mathrm{mg} / 0.75 \mathrm{mg} / 1 \mathrm{mg})(\text { day } 1-29)+\text { ATV } 400 \mathrm{mg} \\
\text { daily (day 16-29) }\end{array}$ \\
\hline & & (i) $\mathrm{EE} C_{\max }$ increased $15 \%(0.99-1.32)$ \\
\hline & & (ii) EE AUC increased 48\% (1.31-1.68) \\
\hline & $N=19$ & (iii) $\mathrm{EE} C_{\min }$ increased $91 \%(1.57-2.33)$ \\
\hline & & (iv) NET $C_{\max }$ increased 67\% (1.42-1.96) \\
\hline & & (v) NET AUC increased 110\% (1.68-2.62) \\
\hline & & (vi) NET $C_{\min }$ increased $362 \%(2.57-5.09)$ \\
\hline & & $\begin{array}{l}\text { EE } 35 \mathrm{mcg} / \mathrm{NGM}(0.18 \mathrm{mg} / 0.215 \mathrm{mg} / 0.25 \mathrm{mg})(\text { day } 1-28) \text {, then EE } \\
25 \mathrm{mcg} / \mathrm{NGM}(0.18 \mathrm{mg} / 0.215 \mathrm{mg} / 0.25 \mathrm{mg})(\text { day } 29-42)+\mathrm{ATV} / \mathrm{r} \\
300 / 100 \text { daily (day 29-42) }\end{array}$ \\
\hline & & (i) $\mathrm{EE} C_{\max }$ decreased $16 \%(0.74-0.95)$ \\
\hline & $N=14$ & (ii) EE AUC decreased 19\% (0.75-0.87) \\
\hline & & (iii) $\mathrm{EE} C_{\min }$ decreased $37 \%(0.55-0.71)$ \\
\hline & & (iv) 17 -deacetyl norgestimate $C_{\max }$ increased $68 \%(1.51-1.88)$ \\
\hline & & (v) 17-deacetyl norgestimate AUC increased 85\% (1.67-2.05) \\
\hline & & (vi) 17 -deacetyl norgestimate $C_{\min }$ increased $102 \%(1.77-2.31)$ \\
\hline
\end{tabular}

Nelfinavir (Viracept)

Protease inhibitor
Viracept package label

[13]

$$
N=12
$$

EE $35 \mathrm{mcg} / \mathrm{NET} 0.4 \mathrm{mg}$ (day 1-15) + NFV $750 \mathrm{mg}$ q $8 \mathrm{~h}$ for 7 days

(i) Decreased EE $C_{\max }$ by $28 \%$

(ii) Decreased EE AUC by $47 \%$

(iii) Decreased $\mathrm{EE} C_{\min }$ by $62 \%$

(iv) No effect on NET $C_{\max }$

(v) Decreased NET AUC by $18 \%$

(vi) Decreased NET $C_{\min }$ by $46 \%$
Lopinavir/ritonavir (Kaletra)

Protease inhibitor
Kaletra package label

[14]

EE 35 mcg/NET 1 mg po daily (21 days) + LPV/r 400/100 po bid (14 days)

(i) $\mathrm{EE} C_{\max }$ decreased $41 \%(0.52-0.66)$

(ii) EE AUC decreased $42 \%(0.54-0.62)$

$N=12$

Vogler et al. [15] (iii) EE $C_{\min }$ decreased $58 \%(0.36-0.49)$

(iv) NET $C_{\max }$ decreased $16 \%(0.75-0.94)$

(v) NET AUC decreased 17\% (0.73-0.94)

(vi) NET $C_{\min }$ decreased $32 \%(0.54-0.85)$

EE $35 \mathrm{mcg} / \mathrm{NET} 1 \mathrm{mg}$ po (day 1 )

EE/NGM patch (day 3-24, new patch every 7 days) + LPV/r (400/100 bid) and stable NRTIs (treatment arm) or no ARV or NRTIs only (control arm)

(i) $\mathrm{COC}$

(a) EE AUC decreased 55\% $(P=0.003)$

(b) EE $C_{48}$ decreased $76 \%(P=0.023)$ 
TABle 2: Continued.

\begin{tabular}{|c|c|c|}
\hline $\begin{array}{l}\text { Drug } \\
\text { Subclass }\end{array}$ & $\begin{array}{c}\text { Source and number of } \\
\text { patients }\end{array}$ & Effect on hormonal contraceptives \\
\hline & $\begin{array}{c}N=8(\text { treatment arm }) \\
N=24(\text { control arm })\end{array}$ & (ii) Patch \\
\hline & & (a) EE AUC decreased 45\% $(P=0.064)$ \\
\hline & & (b) EE $C_{\min }$ decreased $28 \%(P=0.395)$ \\
\hline & & (c) NGMN AUC increased $83 \%(P=0.036)$ \\
\hline & & (d) NGMN $C_{\min }$ increased $134 \%(P=0.036)$ \\
\hline
\end{tabular}

Ritonavir (Norvir)

Protease inhibitor
Ouellet et al. [16]

Day 1: EE $50 \mathrm{mcg} /$ ethynodiol diacetate $1 \mathrm{mg}$

Day 15: RTV 300 mg q $12 \mathrm{~h}$

Day 16: RTV 400 mg q $12 \mathrm{~h}$

$N=23$

Viramune package label [17]

Nevirapine (Viramune) NNRTI

$$
N=32
$$

Mildvan et al. [18]

$$
N=10
$$

Days 17-30: RTV $500 \mathrm{mg} \mathrm{q} 12 \mathrm{~h}$ Ratio of means with $95 \%$ CI

(i) $E E C_{\max }$ decreased $32 \%(0.612-0.758, P<0.001)$

(iii) Ethynodiol diacetate levels not measured days

(i) $\mathrm{EE} C_{\max }$-no change

(ii) EE AUC decreased 20\%

(iii) $\mathrm{EE} C_{\min }$ undetectable

(iv) NET $C_{\max }$ decreased $16 \%$

(v) NET AUC decreased 19\%

(vi) NET $C_{\min }$ undetectable then $200 \mathrm{mg}$ po bid $\times 14$ days

(i) No change in DMPA AUC, $C_{\max }$, or $C_{\min }$

Day 0: EE $35 \mathrm{mcg} / \mathrm{NET} 1 \mathrm{mg}$

Day 2-15: NVP $200 \mathrm{mg}$ po daily

Day 16-29: NVP $200 \mathrm{mg}$ bid

Day 30: NVP $200 \mathrm{mg}$ bid + EE $35 \mathrm{mcg} / \mathrm{NET} 1 \mathrm{mg}$

(i) EE $C_{\max }$ decreased $2 \%(0.78-1.17, P=0.770)$

Day 29: 2nd dose of EE $50 \mathrm{mcg} /$ ethynodiol diacetate $1 \mathrm{mg}$

(ii) EE AUC decreased $40 \%(0.506-0.694, P<0.001)$

EE $35 \mathrm{mcg} / \mathrm{NET} 1 \mathrm{mg}+\mathrm{NVP} 200 \mathrm{mg}$ daily $\times 14$ days, then bid $\times 14$

DMPA 150 mg IM q 3 months + NVP 200 mg po daily $\times 14$ days,

(ii) EE AUC decreased $22 \%(0.54-1.02, P=0.014)$

(iii) EE $t_{1 / 2}(12.5 \pm 3.8 \mathrm{hrs}$ versus $16.9 \pm 4.8 \mathrm{hrs}, P=0.010)$

(iv) NET $C_{\max }$ decreased $14 \%(0.72-1.01, P=0.123)$

(v) NET AUC decreased 18\% $(0.67-0.96, P=0.016)$

(vi) NET $t_{1 / 2} 11.5 \pm 3.7 \mathrm{hrs}$ versus $12.3 \pm 5.3 \mathrm{hrs}(P=0.389)$

Efavirenz (Sustiva) NNRTI
Sustiva package label

[19]

EE $35 \mathrm{mcg} / \mathrm{NGM} 0.25 \mathrm{mg} \times 14$ days + EFV $600 \mathrm{mg}$ daily $\times 14$ days

(i) No change in $\mathrm{EE} C_{\max }, \mathrm{AUC}, C_{\min }$

(ii) NGMN $C_{\max }$ decreased $46 \%(39 \%-52 \%)$

(iii) NGMN AUC decreased 64\% (62\%-67\%)

$N=21$

(iv) NGMN $C_{\min }$ decreased $82 \%(79 \%-85 \%)$

(v) LNG $C_{\max }$ decreased $80 \%(77 \%-83 \%)$ 
Table 2: Continued.

\begin{tabular}{|c|c|c|}
\hline $\begin{array}{l}\text { Drug } \\
\text { Subclass }\end{array}$ & $\begin{array}{l}\text { Source and number of } \\
\text { patients }\end{array}$ & Effect on hormonal contraceptives \\
\hline & \multirow{7}{*}{ Sevinsky et al. [20] } & (vi) LNG AUC decreased 83\% (79\%-87\%) \\
\hline & & (vii) LNG $C_{\min }$ decreased $86 \%(80 \%-90 \%)$ \\
\hline & & ENG implant: decreases ENG (no data provided) \\
\hline & & \\
\hline & & $\begin{array}{l}\text { Cycle 1: EE } 25 \mathrm{mcg} / \mathrm{NGM} 0.18 \mathrm{mg} \text { (day } 1-7), 0.215 \mathrm{mg} \text { (day 8-14), } \\
0.25 \mathrm{mg} \text { (day } 15-21 \text { ) }\end{array}$ \\
\hline & & Cycle 2: EE 35 mcg/NGM 0.25 mg (day 22-56) \\
\hline & & $\begin{array}{l}\text { Cycle 3: EE } 35 \text { mcg/NGM } 0.25 \text { mg (day 57-77) + EFV } 600 \text { mg daily } \\
\text { (day 57-70) }\end{array}$ \\
\hline & \multirow{6}{*}{$N=28$} & (i) $\mathrm{EE} C_{\max }$ increased 6\% (0.95-1.19) \\
\hline & & (ii) EE AUC decreased 10\% (0.80-1.01) \\
\hline & & (iii) $\mathrm{EE} C_{\min }$ decreased $8 \%(0.75-1.14)$ \\
\hline & & (iv) NGMN $C_{\max }$ decreased $46 \%(0.48-0.61)$ \\
\hline & & (v) NGMN AUC decreased 64\% (0.33-0.38) \\
\hline & & (vi) NGMN $C_{\min }$ decreased $82 \%(0.15-0.21)$ \\
\hline \multirow[t]{4}{*}{$\begin{array}{l}\text { Tenofovir (Viread) } \\
\text { NRTI }\end{array}$} & $\begin{array}{l}\text { Viread package label } \\
{[17]}\end{array}$ & \\
\hline & & $\mathrm{EE} 35 \mathrm{mcg} / \mathrm{NGM} 0.18 \mathrm{mg}+\mathrm{TDF}$ \\
\hline & $N=20$ & (i) No change in EE $C_{\max }$, AUC, $C_{\min }$ \\
\hline & & (ii) No change in NGM $C_{\max }, \mathrm{AUC}, C_{\min }$ \\
\hline \multirow[t]{17}{*}{$\begin{array}{l}\text { Etravirine (Intelence) } \\
\text { NNRTI }\end{array}$} & $\begin{array}{l}\text { Intelence package label } \\
\qquad[21]\end{array}$ & \\
\hline & & EE $35 \mathrm{mcg} / \mathrm{NET} 1 \mathrm{mg}$ po daily + ETR $200 \mathrm{mg}$ po bid \\
\hline & & (i) $\mathrm{EE} C_{\max }$ increased $33 \%(1.21-1.46)$ \\
\hline & & (ii) EE AUC increased 22\% (1.13-1.31) \\
\hline & $N=16$ & (iii) $\mathrm{EE} C_{\min }$ increased 9\% (1.01-1.18) \\
\hline & & (iv) NET $C_{\max }$ increased 5\% (0.98-1.12) \\
\hline & & (v) NET AUC decreased 5\% (0.90-0.99) \\
\hline & & (vi) NET $C_{\min }$ decreased 22\% (0.68-0.90) \\
\hline & $\begin{array}{l}\text { Schöller-Gyüre et al. } \\
\qquad[22]\end{array}$ & \\
\hline & & Days 1-21: EE $35 \mathrm{mcg} / \mathrm{NET} 1 \mathrm{mg}$ po daily \\
\hline & & Days 1-15: ETR $200 \mathrm{mg}$ po bid \\
\hline & & (i) $\mathrm{EE} C_{\max }$ increased $33 \%(1.21-1.46)$ \\
\hline & 2 & (ii) EE AUC increased 22\% (1.13-1.31) \\
\hline & $1 v-24$ & (iii) $\mathrm{EE} C_{\min }$ increased 9\% (1.01-1.18) \\
\hline & & (iv) NET $C_{\max }$ increased 5\% $(0.98-1.12)$ \\
\hline & & (v) NET AUC decreased 5\% (0.90-0.99) \\
\hline & & (vi) NET $C_{\min }$ decreased 22\% $(0.68-0.90)$ \\
\hline
\end{tabular}

Raltegravir (Isentress)

Integrase inhibitor
Anderson et al. [23]

EE $35 \mathrm{mcg} / \mathrm{NGM} 0.18 \mathrm{mg} / 0.215 \mathrm{mg} / 0.25 \mathrm{mg}$ po daily + RAL $400 \mathrm{mg}$ po bid or placebo (day 1-21)

(i) EE $C_{\max }$ increased $6 \%(0.98-1.14, P=0.2351)$

$N=19$ (ii) EE AUC decreased $2 \%(0.93-1.04, P=0.5843)$

(iii) NGMN AUC increased 14\% (1.008-1.21, $P=0.0011)$

(iv) NGMN $C_{\max }$ increased $29 \%(1.23-1.37, P<0.0001)$ 
TABle 2: Continued.

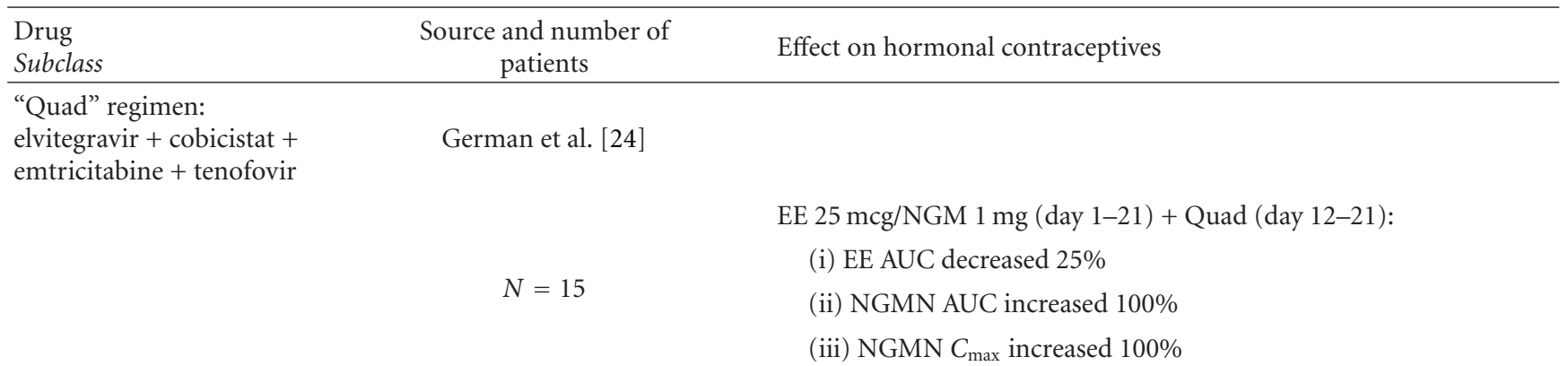

Depot

medroxyprogesterone

acetate (Depo-Provera)

Cohn et al. [25]

Injectable progesterone

Nanda et al. [26]

Group A (control)—no PI or NNRTIs

Group B-NFV + NRTIs

Group C-EFV + NRTIs

Group D-NVP + NRTIs

$N=70$

(i) All received DMPA on day 1 . PK samples were drawn day 0 and after 4 weeks.

(ii) No change in MPA $C_{\max }$, AUC, $C_{\min }$, or terminal half-life when coadministered with HAART regimens containing nelfinavir, efavirenz, or nevirapine

(iii) MPA $C_{\max }$ range (including all groups): $0.32-3.7 \mathrm{ng} / \mathrm{mL}$

(iv) MPA $C_{\min }$ range (including all groups): $0.04-1.31 \mathrm{ng} / \mathrm{mL}$

(v) MPA $\mathrm{AUC}_{0-12}$ values are not reported

Treatment group: 15 women on AZT/3TC/EFV

Control group: 15 HIV+ women not on HAART

Both groups received DMPA $150 \mathrm{mg}$ IM on day 1 and had serum drawn every 2 weeks for 12 weeks total to assess MPA and serum progesterone levels

$$
N=30
$$

(i) DMPA $\mathrm{AUC}_{0-84 \mathrm{~d}}$ increased 1\% (0.85-1.20)

(ii) DMPA $C_{\max }$ increased $1 \%(0.84-1.22)$

(iii) DMPA $C_{\min }$ decreased 10\% $(0.77-1.06)$

Etonogestrel implant

(Implanon)

Progesterone implant
Matiluko et al. [27]

$$
N=1
$$

Lakhi and Govind [28]$$
N=2
$$

Month 0: implant placed

Month 13: HIV diagnosed, started AZT/3TC/EFV

Month 16: diagnosed with ruptured ectopic pregnancy

\section{Patient 1}

(i) July 2004: implant placed.

(ii) January 2007: started EFV/FTC/TDF

(iii) May 2007: diagnosed with intrauterine pregnancy

Patient 2

(i) Conceived with implant in place after starting EFV/LPV (no timeline provided) 
TABle 2: Continued.

\begin{tabular}{|c|c|c|}
\hline $\begin{array}{l}\text { Drug } \\
\text { Subclass }\end{array}$ & $\begin{array}{l}\text { Source and number of } \\
\text { patients }\end{array}$ & Effect on hormonal contraceptives \\
\hline \multicolumn{3}{|c|}{ McCarty et al. [29] } \\
\hline & & June 2005: diagnosed with HIV \\
\hline & & August 2005: started AZT/3TC/EFV \\
\hline & $N=1$ & November 2005: implant placed \\
\hline & & April 2008: diagnosed with right ectopic pregnancy \\
\hline & & January 2009: diagnosed with left ectopic pregnancy \\
\hline \multicolumn{3}{|c|}{ Leticee et al. [30] } \\
\hline & & Patient 1 \\
\hline & & (i) November 2002: started AZT/3TC/EFV \\
\hline & & (ii) January 2004: implant placed \\
\hline & & $\begin{array}{l}\text { (iii) April 2006: diagnosed with intrauterine pregnancy, with } \\
\text { conception estimated in Dec. } 2005 \text { based on ultrasound (23 } \\
\text { months after implant placement) }\end{array}$ \\
\hline & \multirow[t]{5}{*}{$N=2$} & Patient 2 \\
\hline & & (i) 2001: HIV diagnosed \\
\hline & & (ii) July 2005: implant placed \\
\hline & & (iii) April 2007: started on EFV/TDF/FTC \\
\hline & & (iv) October 2007: pregnant after condom rupture \\
\hline
\end{tabular}

Levonorgestrel Intrauterine system (Mirena)

Progesterone IUD

$$
N=12
$$

Lehtovirta et al. [32]

$$
N=6
$$

Heikinheimo et al. [33]

$$
N=40
$$

LNG-IUS placed between cycle day $1-7$. Serum drawn immediately before LNG-IUS insertion and at 1 week, 3 months, 6 months, and 12 months. No difference in serum LNG levels in HIV-positive women on HAART compared to HIV-positive women not on HAART (data presented graphically), and consistent with HIV negative historical controls

Retrospective review of 6 HIV-positive women with LNG-IUS. Two were treated with HAART, and 4 were on no ARVs. Mean duration of LNG-IUS use $=45$ months (range 12-72 months). No PK assessments were performed. No pregnancies or adverse events were reported

Case-control study of 15 HIV-positive women using LNG-IUS and 25 HIV-positive women not using LNG-IUS was conducted. 54\% of LNG-IUS users and $56 \%$ of controls were on HAART at beginning of followup, $73 \%$ and $76 \%$ were on HAART at the end of followup. No $\mathrm{PK}$ assessments were performed. No pregnancies and no differences in CD4 counts or HIV VL were seen between the two groups

Levonorgestrel emergency contraception (Plan B)

Carten et al. [34]

Day 1: LNG 0.75 mg orally, PK blood sampling immediately before and for 12 hours after LNG dose

Days 4-17: EFV $600 \mathrm{mg}$ qhs

Day 18: LNG $0.75 \mathrm{mg}$ orally, PK blood sampling immediately before

$$
N=24
$$
and for 12 hours after LNG dose

(i) LNG AUC $_{0-12 \mathrm{~h}}$ decreased $48 \%(0.36-0.48, P<0.0001)$

(ii) LNG $C_{\max }$ decreased $45 \%(0.49-0.63, P<0.0001)$

(iii) LNG $C_{\min }$ decreased 69\% $(0.26-0.36, P<0.0001)$

Abbreviations. EE: ethinyl estradiol, NET: norethindrone, NGM: norgestimate, NGMN: norelgestromin, LNG: levonorgestrel, ENG: etonogestrel, DMPA: depot medroxyprogesterone acetate, MPA: medroxyprogesterone acetate, $C_{\max }$ : maximum serum concentration, AUC: area under the concentrationtime curve, $C_{\min }$ : minimum serum concentration, $t_{1 / 2}$ : half-life, ATV: atazanavir, NFV: nelfinavir, LPV/r: lopinavir/ritonavir, NVP: nevirapine, ATV/r: atazanavir/ritonavir, EFV: efavirenz, AZT: zidovudine, 3TC: lamivudine, FTC: emtricitabine, TDF: tenofovir, LPV: lopinavir, ETR: etravirine. 
more reassuring data, COCs should not be considered as a primary method of contraception in HIV-positive women taking nevirapine [18].

An open-label study of interactions between saquinavir and a low-dose COC containing EE/gestodene showed no effect on the pharmacokinetics of saquinavir [46]. Pharmacokinetic parameters for EE and gestodene were not assessed in this study, so it is unclear whether saquinavir impacts the efficacy of COCs.

The potential interactions between maraviroc (a CCR5 coreceptor antagonist, which blocks the ability of the HIV virus to enter a cell) and a COC were investigated in a double-blind, placebo-controlled, crossover study. Fifteen healthy HIV-negative women were given daily doses of EE/levonorgestrel (LNG) and either maraviroc (100 mg twice daily) or placebo [47]. There was no significant difference in the AUC or $C_{\max }$ for EE, LNG, or maraviroc between the two study arms, indicating that maraviroc has no effect on the pharmacokinetics of these hormones.

Schöller-Gyüre et al. studied EE/NET and concomitant use of etravirine (an NNRTI) in healthy HIV-negative women [22]. Participants took COC for two consecutive cycles ( 21 days of active pills followed by a pill-free week), then added etravirine during the first 15 days of cycle 3. Pharmacokinetic assessments performed on day 15 of COC cycles 2 and 3 revealed a 22\% increase in EE AUC, a 33\% increase in $\mathrm{EE} C_{\max }$, and a $9 \%$ increase in $\mathrm{EE} C_{\min }$ when the COC was given with etravirine, while norethindrone had a $22 \%$ decrease in $C_{\min }$ but exhibited no change in AUC or $C_{\max }$. Serum levels of $\mathrm{LH}, \mathrm{FSH}$, and progesterone were also measured on days 1 and 14 of cycle 3 . There were no significant changes in any of these levels when etravirine was added compared to when the COC was taken alone. There was no serologic evidence of ovulation in any of the study participants. Ultimately, the authors concluded that COC could be used safely by women taking etravirine as their contraceptive efficacy appears to be maintained [22].

A recent study evaluated the effect of the PIs lopinavir and ritonavir on the pharmacokinetics of orally and transdermally administered hormonal contraceptives in HIVinfected women [15]. Participants in the treatment arm were taking a stable lopinavir/ritonavir dose in combination with dual NRTI therapy, while those in the control arm were taking NRTIs only or no ARV therapy at all. Women in the treatment and control arms all received a single dose of a COC containing EE/NET five to seven days after the start of menses, followed by serial blood samples. Participants applied the contraceptive patch (EE/norelgestromin) 48 hours after taking the single-dose COC, and serial blood sampling for PK parameters and progesterone was performed. There was a 55\% decrease in EE AUC $(P=$ 0.003 ) and a $76 \%$ decrease in serum EE concentration 48 hours after COC ingestion $(P=0.023)$ in the PI treatment arm compared to controls. (Results for the contraceptive patch in this study are discussed below.) Norelgestromin levels were not assessed in patients taking the COC, making any conclusions about COC contraceptive efficacy when coadministrated with lopinavir/ritonavir impossible.
Raltegravir is an HIV integrase strand transfer inhibitor which prevents the integration of HIV DNA into the host genome; it is not known to affect the activity of any CYP isoenzymes [48]. A randomized two-arm crossover study investigating interactions between raltegravir and a triphasic COC containing EE/norgestimate found no difference in $\mathrm{EE}$ PK parameters between the two groups. There were small decreases in the $C_{\min }$ and AUC of norelgestromin, the active metabolite of norgestimate, but these were not felt to be clinically significant [23].

Efavirenz is an NNRTI that is a potent inhibitor of CYP3A4 as well as a known teratogen [19]. Because efavirenz has been associated with neural tube defects, especially when exposure occurs in the first trimester, effective contraception is particularly important for HIV-positive women taking this drug. A study of healthy HIV-negative women evaluated the pharmacokinetic interaction between oncedaily efavirenz and a COC containing EE/norgestimate [20]. Participants completed one 28-day cycle with a $25 \mathrm{mcg}$ EE/triphasic norgestimate COC. All participants who had acceptable baseline safety evaluations during this first cycle took a higher-dose $(35 \mathrm{mcg}) \mathrm{EE} / \mathrm{monophasic}$ norgestimate $(0.25 \mathrm{mg}) \mathrm{COC}$ for cycles 2 and 3, with 14 days of efavirenz taken during cycle 3 (days 57-70). Pharmacokinetic blood samples for EE (days 14, 42, and 70), norelgestromin (the active metabolite of norgestimate; days 42 and 70), and efavirenz (day 70) were drawn throughout the study. There was no difference in EE AUC between cycle 2 (35 mcg EE/0.25 mg norgestimate) and cycle 3 (35 mcg $\mathrm{EE} / 0.25 \mathrm{mg}$ norgestimate $+600 \mathrm{mg}$ efavirenz), while there was a significant decrease in norelgestromin $C_{\max }(46 \%)$, AUC $(64 \%)$, and $C_{\min }(82 \%)$ in cycle 3 compared to cycle 2. Despite the decrease in norelgestromin concentrations, serum progesterone levels remained low throughout all cycles, suggesting that ovulation was successfully suppressed during efavirenz administration.

Many ARVs are now formulated in combination pills to facilitate adherence with therapy. A new example of such a formulation is the "Quad" regimen, which contains elvitegravir (an investigational integrase inhibitor), cobicistat (an investigational pharmacoenhancer that has no antiretroviral activity itself, but is used to inhibit elvitegravir metabolism), and a common NRTI backbone of emtricitabine and tenofovir. In a Phase 1, open-label, fixed sequence study, 15 HIV-negative healthy women were given a COC containing $\mathrm{EE} /$ norgestimate for two 28-day cycles [24]. On days 12 to 21 of the second cycle, they were also given the Quad regimen. Serum sampling for EE and norelgestromin PK parameters was performed on day 21 of each cycle, and samples for PK of elvitegravir and cobicistat were performed on day 21 of the second cycle only. While there was a $25 \%$ decrease in EE AUC from the first to the second cycle, the AUC and $C_{\max }$ of norelgestromin doubled. There was no change in serum progesterone or FSH levels measured on day 21 of the treatment cycle compared to day 21 of the baseline cycle. Levels of LH were lower when the COC was given with the Quad regimen compared to the COC alone. Ultimately, the authors concluded that the contraceptive efficacy of the COC was maintained, but recommended that a COC with at least 
$30 \mathrm{mcg}$ EE is considered in women taking the Quad regimen to offset the decreased EE levels noted when the two were taken together.

The CDC MEC lists all combined hormonal contraceptive methods (COC, patch, and vaginal ring) as Category 1 for women with HIV or AIDS [35]. In a separate discussion of drug interactions between hormonal contraceptives and ARVs, it is noted that some NNRTIs and ritonavir-boosted PIs are associated with decreased levels of contraceptive hormones that could compromise contraceptive effectiveness. Consequently, the CDC lists combined hormonal methods as Category 2 for women taking NNRTIs, and Category 3 for women taking ritonavir-boosted PIs. The lack of data regarding clinical outcomes such as escape ovulation or unintended pregnancy makes interpretation of these findings and determination of their clinical significance difficult.

3.1.2. Contraceptive Patch and Contraceptive Vaginal Ring. The only study that has investigated the contraceptive patch in women taking ARV is the AIDS Clinical Trial Group (ACTG) Protocol A5188 study, first discussed above and which evaluated the pharmacokinetic interactions between lopinavir/ritonavir and the transdermal contraceptive patch (EE/norelgestromin) in HIV-positive women [15]. Compared to women not taking protease inhibitors, serum of women taking lopinavir/ritonavir demonstrated a $45 \%$ decrease in the AUC of $\mathrm{EE}(P=0.064)$. There was also an $83 \%$ increase in AUC of norelgestromin in the treatment (lopinavir/ritonavir) arm compared to controls. This decrease in EE was similar to that seen when a single dose of oral COC was given, suggesting that the PI interaction with the hormonal contraception is mediated thorough the liver rather than enzymes within the gut. Only eight women on lopinavir/ritonavir were evaluated in this study, and no data are available regarding adverse events or contraceptive failure. Progesterone levels were decreased in both treatment and control arms, suggesting continued ovulation suppression despite changes in hormone levels.

No studies evaluating the pharmacokinetics of the contraceptive intravaginal ring in the setting of ARV use were identified. The package label for NuvaRing (which releases $15 \mathrm{mcg}$ EE/120 mcg etonogestrel (ENG) daily) refers to interactions identified between COCs and protease inhibitors that may affect contraceptive efficacy [49]. There is no discussion of how the pharmacokinetics of a vaginally administered hormone might differ from one that is orally administered, and there are no data specific to etonogestrel. As the influence of ARVs on contraceptive steroids seems to be related to their metabolism rather than absorption, there may be no reason to expect different results between vaginal and oral administration. In other words, while the effects of firstpass metabolism are avoided by nonoral administration, the hepatic clearance of the drug from the serum may be the more important site of drug interaction.

As noted above, the CDC MEC recommendations for combined hormonal methods include COCs, patch, and ring, as there are few studies that offer data for the nonoral combined methods [35]. All combined methods are considered Category 1 for women with HIV or AIDS,
Category 2 for women taking NNRTIs, and Category 3 for women taking ritonavir-boosted PIs.

\subsection{Progestin-Only Methods}

3.2.1. Progestin-Only Pills (POPs). The effectiveness of progestin-only pills is known to be reduced by hepatic enzyme-inducing drugs [50]. POPs are used by only a minority of women, and there are no published studies on the interaction between POPs and ARVs. Since POPs available in the US contain NET, and those available elsewhere contain LNG or desogestrel (DSG), one could potentially extrapolate data from studies with COCs containing these hormones. The CDC assigns the same recommendation categories to POPs as to COCs; that is, concomitant use of POP with NRTIs is Category 1, with NNRTIs is Category 2, and with ritonavir-boosted PIs is Category 3 [35].

3.2.2. Depot Medroxyprogesterone Acetate (DMPA). The most recent labeling for depot medroxyprogesterone acetate (DMPA, Depo-Provera) does not mention any interaction with protease inhibitors or other antiretroviral drugs [51]. The ACTG Protocol A5093 evaluated the pharmacodynamics and safety profile of DMPA in $70 \mathrm{HIV}$-positive women on stable ARV regimens [52]. All women in the study were taking at least two NRTIs in addition to an NNRTI (efavirenz or nevirapine) or a PI (nelfinavir). Pharmacokinetic parameters of DMPA were not assessed, but no participants ovulated nor became pregnant over the 12-week study period.

An open-label steady-state pharmacokinetic study evaluated the interactions between DMPA and selected ARVs [25]. Study participants were HIV-positive women on one of four ARV regimens: group A-NRTIs only or no ARVs (no NNRTI or PI); group B-nelfinavir and NRTIs; group $\mathrm{C}$-efavirenz and NRTIs; and group D-nevirapine and NRTIs. Serum samples for medroxyprogesterone acetate (MPA) were measured immediately prior to DMPA injection and 4 weeks after the injection, while serum progesterone concentrations were measured immediately prior to DMPA injection and every 2 weeks throughout the 12 -week study period. There were no alterations in the pharmacokinetic profile of DMPA noted in any of the treatment groups. There were no pregnancies during the study, and all participants had low $(<5 \mathrm{ng} / \mathrm{mL})$ serum progesterone levels, consistent with ovulation suppression [25].

Another prospective study compared the pharmacokinetics of DMPA among $15 \mathrm{HIV}$-positive women on HAART (zidovudine, lamivudine, and efavirenz) with $15 \mathrm{HIV}$ positive women on no ARVs [26]. Serum DMPA and progesterone levels were drawn every 2 weeks throughout the 12-week study. In both groups, the MPA $C_{\max }$ was reached within 14 days of the DMPA injection, and there were no differences in MPA AUC, $C_{\min }$, half-life, or bleeding patterns between the two groups. The authors concluded that there is no need to shorten the interval between DMPA injections in women taking ARVs, as there is no evidence of decreased serum levels of MPA in women on HAART. 
For women with HIV or AIDS, on any ARV regimen, the CDC MEC considers DMPA use to be Category 1 (no restriction for use of contraceptive method) [35].

3.2.3. Contraceptive Implant. There are several case reports that describe contraceptive failure of Implanon, a 3-year etonogestrel implant that releases $20-30 \mathrm{mcg}$ ENG per day, among HIV-positive women using ARVs. A young woman was diagnosed with HIV 13 months after placement of the implant, at which time she started a HAART regimen of zidovudine, lamivudine, and efavirenz [27]. Sixteen months later she was diagnosed with a ruptured ectopic pregnancy. The implant was palpable in her arm at the time of her pregnancy, suggesting that insertion difficulty was not to blame for its contraceptive failure. No evaluation of serum ENG levels was performed. In a letter to the editor, two additional HIV-positive patients who became pregnant while using Implanon and taking HAART are described [28]. Both became amenorrheic due to the implant, were later changed to ARV regimens that included efavirenz, and were subsequently diagnosed with intrauterine pregnancies. One patient chose to continue the pregnancy and delivered a healthy infant at term, while the other opted for a secondtrimester abortion. Again, no serum levels of ENG or the ARVs are reported for either patient.

In another case report, a 34-year-old HIV-positive woman on a HAART regimen of zidovudine, lamivudine, and efavirenz was treated for a ruptured ectopic pregnancy 28 months after insertion of an etonogestrel contraceptive implant [29]. Interestingly, this patient did not have her Implanon removed and was treated for a subsequent ectopic pregnancy on the contralateral side nine months later (i.e., more than 3 years after initial placement of the implant). A fourth case report describes two different HIV-positive women who conceived intrauterine pregnancies while the etonogestrel implant was in place [30]. One was a 31-yearold HIV-positive women treated with efavirenz, zidovudine and lamivudine. The other was a 32-year-old HIV-positive woman on tenofovir, emtricitabine, and efavirenz. There are no pharmacokinetic data available regarding interactions between etonogestrel and any ARVs, but efavirenz is known to induce hepatic $\mathrm{P} 450$ activity, making it a plausible cause of the implant's contraceptive failure [27].

At this time, the CDC MEC lists Implanon as Category 1 for women with HIV or AIDS, Category 1 for women taking NRTIs, and Category 2 for women taking NNRTIs or ritonavir-boosted PIs [35]. A consistent theme of the above case reports seems to be that pregnancies conceived during ARV use with Implanon in place occur after 24 months of use. Data are currently lacking, but it seems reasonable to consider early replacement of the implant.

3.2.4. Levonorgestrel Intrauterine System (LNG-IUS). As for most nonoral hormonal contraceptives, the labeling for the LNG-IUS (Mirena) cautions against its concomitant use with drugs that induce hepatic P450 activity [53]. The label specifically mentions concern for PIs and NNRTIs potentially altering the serum concentration of progestins.
A 2006 study evaluated serum levels of LNG before and up to twelve months after LNG-IUS placement in a series of twelve HIV-positive women [31]. Ten of the women enrolled $(83 \%)$ were on HAART during the study period. Serum levels of LNG were similar between women with and without HAART and were in the same range as had been reported in HIV-negative women, suggesting no effect of HAART on the absorption or metabolism of LNG when used for intrauterine contraception.

Two studies provide follow-up data on the LNG-IUS in HIV-infected women [32, 33]. Both concluded that it is a safe option for HIV-positive women desiring longterm contraception and those looking for treatment of heavy menstrual bleeding. In the 2011 study, there was no difference in CD4 counts, HIV viral loads, use of ARVs, or pregnancies between the intervention (15 women who received LNG-IUS) and control (25 women using other methods of contraception) groups [33]. No pharmacokinetic evaluation was done as part of these studies.

It should be noted that the primary mechanism of action of the LNG-IUS is unrelated to serum LNG levels. Rather, it is primarily due to a foreign body reaction and direct effects from the locally released progestin. Further, serum LNG levels can vary widely in users of the LNG-IUS, without apparent correlation to contraceptive effect. Therefore, the clinical contraceptive impact of altered serum LNG levels in women using the LNG-IUS may not be significant. The CDC MEC assigns Category 2 (benefits outweigh risks) to the use of any intrauterine contraception (including the LNG-IUS) for women with HIV who are stable on ARV therapy of any kind [35]. Of note, for women with AIDS, whether on ARVs or not, insertion of an IUD is considered to be Category 3 [35].

3.2.5. Emergency Contraception (EC). Two types of dedicated emergency contraceptive (EC) pills exist: progestin-only pills containing levonorgestrel, and antiprogestins (either mifepristone or ulipristal acetate). The Plan B (two oral doses of LNG $0.75 \mathrm{mg}$ taken 12 hours apart within 72 hours of unprotected intercourse) package labeling raises theoretical concerns regarding coadministration with drugs that induce hepatic enzymes, but provides no data to support or refute these concerns [54]. Only one study was identified that evaluated the interaction between LNG EC and efavirenz [34]. In this single-arm, open-label study, $21 \mathrm{HIV}$-negative women were given a single course of Plan B. Serial pharmacokinetic blood samples were drawn immediately before and for 12 hours after the first LNG dose. Participants then took 14 days of EFV (600 mg daily), followed by a second course of Plan $\mathrm{B}$ and blood sampling after the first LNG dose. Compared to baseline, LNG coadministrated with EFV resulted in a 58\% decrease in AUC $(P<0.0001)$ and statistically significant decreases in $C_{\min }, C_{\max }$, and half-life [34]. While the authors acknowledge that the minimum effective concentration of LNG for emergency contraception is unknown, they suggest that women taking EFV may require higher doses of LNG to prevent pregnancy, such as the one-time dose of LNG 1.5 $\mathrm{mg}$. A recent review of EC similarly suggests that an increased progestin dose may be considered if an ARV with known 
effects on contraceptive hormones is used concomitantly [55]. This recommendation is based on an older study and may not apply to all current ARVs. No data exist regarding the use of antiprogestins during ARV therapy.

COCs may also be used in higher doses to provide EC, referred to as the Yuzpe regimen [56]. There are no data specifically assessing interactions between ARVs and COCs in these doses, but one could expect analogous pharmacokinetic effects to those seen after single-dose COC administration. Whether these effects alter the efficacy of the Yuzpe regimen is not known. In general, the Yuzpe method is less effective than progestin or antiprogestin EC. Regardless of type chosen, theoretical concern for decreased effectiveness should not deter providers from offering EC to women with HIV.

\section{Discussion}

Assessing the effects of antiretroviral drugs on the efficacy of hormonal contraceptives is a challenging undertaking for many reasons. Most drug-drug interaction studies aim to investigate only two drugs at a time so as to facilitate interpretation of any changes in pharmacokinetic parameters. Studying antiretroviral drugs individually does not reflect their real-world application as part of multiagent HAART regimens, when there may be multiple layers of enzyme induction or inhibition as well as other physiologic effects that may alter drug absorption or excretion. There are also potential disadvantages to conducting these studies in healthy HIV-negative volunteers, who may be better able to adhere to a particular contraceptive method than HIVpositive women who have to contend with managing their illness as well as following a study protocol.

The concept of "pill burden" may be an additional factor to consider when helping an HIV-positive woman on ARVs to choose the best contraceptive method for her. High pill burden is a barrier to adherence to $\operatorname{HAART}[57,58]$. In this context, a nondaily, nonoral method of contraception may be preferred for some women using ARVs. However, for methods such as the contraceptive patch and vaginal ring there are limited or no data to guide the clinician or the HIVpositive woman.

Of the information that is available about interactions of hormonal contraception and ARVs, much is based on a limited number of PK studies with small sample sizes and short durations of hormone and ARV exposure. Pharmacokinetic measurements are often affected by the hepatic P450 system, but AUC and $C_{\max }$, among other measurements, are dependent on many other factors including age, weight, length of exposure to hormones and ARVs, absorption factors, and even pharmacogenetics. An increase in hormone levels could possibly affect side effects and potential complications (e.g., thromboembolism). A large enough decrease in hormone levels could potentially decrease contraceptive efficacy. Interestingly, among ARVs which lead to decreased hormone levels, the decrease seems to be greatest for EE levels, but it is the progestin component of combined hormonal contraception which is more important to contraceptive efficacy. In addition, therapeutic levels of contraceptive hormones are not actually known. Therefore, the clinical relevance of a percentage decrease in a serum contraceptive hormone level is unclear. A more useful measurement of contraceptive efficacy would be assessments of ovulation via serum hormone levels (e.g., progesterone, $\mathrm{LH}, \mathrm{FSH}$ ) and serial pelvic ultrasounds. With the exception of the Schöller-Gyüre study [22], no markers of ovulation have been included in studies on the interaction of ARVs and hormonal contraception. Therefore, recommendations based on small studies relying only on pharmacokinetic measurements should be treated cautiously.

The development of transdermal and transvaginal dosing regimens avoids the first-pass metabolism that occurs in the gastrointestinal system, thereby reducing the effect of CYP metabolism of contraceptive hormones. Because of this avoidance, nonoral routes of administration would be expected to differ from COCs regarding their metabolism and therefore drug interactions. However, the only study available which looks at a nonoral administration of hormonal contraception with ARVs shows similar changes in hormone pharmacokinetics as studies investigating COCs [22].

From the available evidence, the efficacy of DMPA and LNG-IUS should not be affected by ARV use. Other hormonal methods may be affected by ARV coadministration. Use of condoms in addition to hormonal contraception is recommended. Of course, this recommendation is warranted for all heterosexually active HIV-positive women in order to decrease HIV transmission and acquisition of other sexually transmitted diseases.

The particular case of the ENG implant is intriguing. All reports of contraceptive implant failure reviewed here occurred in HIV-positive women taking efavirenz as a component of their HAART regimen. Based on available information, it appears that all pregnancies that occurred during coadministration of Implanon and efavirenz were conceived in the latter half of the three years for which Implanon is licensed. This raises the question of whether the implant is wholly ineffective when used by women taking efavirenz, or whether its contraceptive activity is depleted more quickly than in women not taking efavirenz. Avoiding unintended pregnancy in women treated with efavirenz is highly important given the potential for teratogenicity with efavirenz exposure, and the implant typically provides excellent, long-acting reversible birth control. Studies that address the mechanism by which efavirenz affects subdermallyimplanted etonogestrel are sorely needed.

The issue of whether hormonal contraceptives are safe for women with HIV has been hotly debated in the literature. The WHO recently published a technical statement regarding hormonal contraceptive use by women with HIV, after performing extensive reviews of the literature regarding effects of hormonal contraception on HIV acquisition, transmission, and disease progression [59]. Despite a recent study that found an increase in both HIV acquisition by uninfected women and HIV transmission by HIV-positive women using DMPA [60], the overall body of evidence does not support a definite association. Consequently, the CDC and WHO continue to list DMPA and other hormonal 
contraceptives as Category 1 for women infected with HIV $[35,61]$.

There are some significant pitfalls in relying on individual drugs' package labeling for guidance regarding drug interactions, real, or theoretical. Clinical trials performed during drug development typically involve small numbers of patients, so certain interactions may not come to light until a licensed drug is used in hundreds or thousands of patients. Postmarketing data is an important source of additional information about a drug once it has been used in the general population, but most drugs do not have active surveillance systems and instead rely on patient and provider self-report. Data collected by manufacturers in pursuit of drug approval may not be published in the peer-reviewed literature, and thus may not be readily accessible. However, when there are no published data to guide health care providers, the drug manufacturers' recommendations can provide some basis for clinical decision-making.

\section{Conclusions}

Although there is no concrete data demonstrating reduced efficacy of the ENG implant when coadministrated with HAART, the numerous case reports of contraceptive failure in women taking efavirenz are concerning. It would be prudent for health care providers to counsel HIV-positive women requesting Implanon regarding the importance of dual contraception, particularly if their HAART regimen includes efavirenz. Until there is a better understanding of whether these medications truly interact, Implanon should continue to be made available to women living with HIV who desire LARC.

While there are demonstrable changes in the serum concentrations of EE and various progestins used in hormonal contraception, it remains unclear how clinically relevant these changes are and whether they can be interpreted as altered contraceptive efficacy. Studies that evaluate clinical markers of ovulation or actual pregnancy rates are necessary to clarify which hormonal contraceptives are most effective for women taking antiretroviral drugs. The data are mixed regarding interactions with combined oral contraceptives, and there is a dearth of information regarding the contraceptive patch, vaginal ring, and implant. However, the data are reassuring that DMPA and the LNG-IUS remain highly effective contraceptives even when used in conjunction with antiretroviral drugs. The Medical Eligibility Criteria published by the CDC and WHO each designate hormonal contraceptives as safe and appropriate (i.e., Category 1) for women with HIV or AIDS, with caution advised for women treated with NNRTIs or ritonavir-boosted PIs $[35,61]$.

\section{References}

[1] UNAIDS/WHO, “AIDS Epidemic Update,” 2009.

[2] H. W. Reynolds, B. Janowitz, R. Homan, and L. Johnson, "The value of contraception to prevent perinatal HIV transmission," Sexually Transmitted Diseases, vol. 33, no. 6, pp. 350-356, 2006.
[3] M. Zieman, R. A. Hatcher, C. Cwiak, P. D. Darney, M. D. Creinin, and H. R. Stosur, A Pocket Guide to Managing Contraception, Bridging the Gap Foundation, Tiger, Ga, USA, 2007.

[4] FFPHRC Guidance, "Drug interactions with hormonal contraception," Journal of Family Planning and Reproductive Health Care, vol. 31, no. 2, pp. 139-150, 2005.

[5] M. W. Van Den Heuvel, A. J. M. Van Bragt, A. K. M. Alnabawy, and M. C. J. Kaptein, "Comparison of ethinylestradiol pharmacokinetics in three hormonal contraceptive formulations: The vaginal ring, the transdermal patch and an oral contraceptive," Contraception, vol. 72, no. 3, pp. 168-174, 2005.

[6] V. B. Mahesh, "The dynamic interaction between steroids and gonadotropins in the mammalian ovulatory cycle," Neuroscience and Biobehavioral Reviews, vol. 9, no. 2, pp. 245-260, 1985.

[7] D. J. Hemrika, E. H. Slaats, J. C. Kennedy, T. J. M. De Vries Robles-Korsen, and J. Schoemaker, "Pulsatile luteinizing hormone secretion during the first and the fourth cycle on two different oral contraceptives containing gestodene," Acta Endocrinologica, vol. 129, no. 3, pp. 229-236, 1993.

[8] L. Speroff and M. Fritz, Clinical Gynecologic Endocrinology and Infertility, Lippincott Williams \& Wilkins, Baltimore, Md, USA, 7th edition, 2005.

[9] F. P. Guengerich, "Metabolism of $17 \alpha$-ethynylestradiol in humans," Life Sciences, vol. 47, no. 22, pp. 1981-1988, 1990.

[10] A. B. Edelman, G. Cherala, and F. Z. Stanczyk, "Metabolism and pharmacokinetics of contraceptive steroids in obese women: a review," Contraception, vol. 82, no. 4, pp. 314-323, 2010.

[11] Panel on Antiretroviral Guidelines for Adults and Adolescents, "Guidelines for the use of antiretroviral agents in HIV-1-infected adults and adolescents," Department of Health and Human Services, 2009, http://www.aidsinfo .nih.gov/contentfiles/lvguidelines/adultandadolescentgl.pdf.

[12] Bristol-Meyers Squibb, Reyataz (atazanavir) Package Label, 2010.

[13] Agouron Pharmaceuticals, Viracept (nelfinavir) Package Label, 2009.

[14] Abbott Laboratories, Kaletra (lopinavir/ritonavir) Package Label, 2010.

[15] M. A. Vogler, K. Patterson, L. Kamemoto et al., "Contraceptive efficacy of oral and transdermal hormones when co-administered with protease inhibitors in HIV-1-infected women: Pharmacokinetic results of ACTG trial A5188," Journal of Acquired Immune Deficiency Syndromes, vol. 55, no. 4, pp. 473-482, 2010.

[16] D. Ouellet, A. Hsu, J. Qian et al., "Effect of ritonavir on the pharmacokinetics of ethinyl oestradiol in healthy female volunteers," British Journal of Clinical Pharmacology, vol. 46, no. 2, pp. 111-116, 1998.

[17] Boehringer Ingelheim Pharmaceuticals, Viramune (nevirapine) Package Label, 2010.

[18] D. Mildvan, R. Yarrish, A. Marshak et al., "Pharmacokinetic interaction between nevirapine and ethinyl estradiol/ norethindrone when administered concurrently to HIVinfected women," Journal of Acquired Immune Deficiency Syndromes, vol. 29, no. 5, pp. 471-477, 2002.

[19] Bristol-Meyers Squibb, Sustiva (efavirenz) Package Label, 2010.

[20] H. Sevinsky, T. Eley, A. Persson et al., "The effect of efavirenz on the pharmacokinetics of an oral contraceptive containing 
ethinyl estradiol and norgestimate in healthy HIV-negative women," Antiviral Therapy, vol. 16, no. 2, pp. 149-156, 2011.

[21] Janssen Pharmaceuticals, Intelence (etravirine) Package Label, 2008.

[22] M. Schöller-Gyüre, T. N. Kakuda, B. Woodfall et al., "Effect of steady-state etravirine on the pharmacokinetics and pharmacodynamics of ethinylestradiol and norethindrone," Contraception, vol. 80, no. 1, pp. 44-52, 2009.

[23] M. S. Anderson, W. D. Hanley, A. R. Moreau et al., "Effect of raltegravir on estradiol and norgestimate plasma pharmacokinetics following oral contraceptive administration in healthy women," British Journal of Clinical Pharmacology, vol. 71, no. 4, pp. 616-620, 2011.

[24] P. German, M. Wang, D. Warren, and B. P. Kearney, "Pharmacokinetic interaction between Norgestimate/Ethinyl Estradiol and EVG/COBI/FTC/TDF single tablet regimen," in Proceedings of the 12th International Workshop on Clinical Pharmacology of HIV Therapy, Miami, Fla, USA, April 2011.

[25] S. E. Cohn, J. G. Park, D. H. Watts et al., "Depo-medroxyprogesterone in women on antiretroviral therapy: Effective contraception and lack of clinically significant interactions," Clinical Pharmacology and Therapeutics, vol. 81, no. 2, pp. 222-227, 2007.

[26] K. Nanda, E. Amaral, M. Hays, M. A. M. Viscola, N. Mehta, and L. Bahamondes, "Pharmacokinetic interactions between depot medroxyprogesterone acetate and combination antiretroviral therapy," Fertility and Sterility, vol. 90, no. 4, pp. 965-971, 2008.

[27] A. A. Matiluko, L. Soundararjan, and P. Hogston, "Early contraceptive failure of Implanon in an HIV-seropositive patient on triple antiretroviral therapy with zidovudine, lamivudine and efavirenz," Journal of Family Planning and Reproductive Health Care, vol. 33, no. 4, pp. 277-278, 2007.

[28] N. Lakhi and A. Govind, "Implanon failure in patients on antiretroviral medication: the importance of disclosure," Journal of Family Planning and Reproductive Health Care, vol. 36, no. 3, pp. 181-182, 2010.

[29] E. J. McCarty, H. Keane, K. Quinn, and S. Quah, "Implanonw failure in an HIV-positive woman on antiretroviral therapy resulting in two ectopic pregnancies," International Journal of STD and AIDS, vol. 22, no. 7, pp. 413-414, 2011.

[30] N. Leticee, J.-P. Viard, A. Yamgnane, M. Karmochkine, and A. Benachi, "Contraceptive failure of etonogestrel implant in patients treated with antiretrovirals including efavirenz," Contraception, vol. 85, no. 4, pp. 425-427, 2012.

[31] O. Heikinheimo, P. Lehtovirta, J. Suni, and J. Paavonen, "The levonorgestrel-releasing intrauterine system (LNG-IUS) in HIV-infected women-effects on bleeding patterns, ovarian function and genital shedding of HIV," Human Reproduction, vol. 21, no. 11, pp. 2857-2861, 2006.

[32] P. Lehtovirta, J. Paavonen, and O. Heikinheimo, "Experience with the levonorgestrel-releasing intrauterine system among HIV-infected women," Contraception, vol. 75, no. 1, pp. 3739, 2007.

[33] O. Heikinheimo, P. Lehtovirta, I. Aho, M. Ristola, and J. Paavonen, "The levonorgestrel-releasing intrauterine system in human immunodeficiency virusinfected women: Z 5year follow-up study," American Journal of Obstetrics and Gynecology, vol. 204, no. 2, pp. 126.e1-126.e4, 2011.

[34] M. L. Carten, J. Kiser, A. Kwara, S. MaWhinney, and S. $\mathrm{Cu}$-Uvin, "Pharmacokinetic (PK) interactions between the hormonal emergency contraception Plan B (levonorgestrel) and efavirenz (EFV)," in Proceedings of the 17th Conference on Retroviruses and Opportunistic Infections, San Francisco, Calif, USA, February 2010.

[35] Department of Health and Human Services Centers for Disease Control and Prevention, "US medical eligibility criteria for contraceptive use," Morbidity and Mortality Weekly Report, vol. 59, no. 4, pp. 1-86, 2010.

[36] Wyeth Laboratories. LoOvral (ethinyl estradiol/norgestrel) Package label, 2007.

[37] Wyeth Pharmaceuticals, Alesse (ethinyl estradiol/levonorgestrel) Package Label, 2008.

[38] Ortho-McNeil-Janssen Pharmaceuticals, Ortho TriCyclen (ethinyl estradiol/norgestimate) Package Label, 2009.

[39] Bayer HealthCare Pharmaceuticals, Yaz (ethinyl estradiol/ drospirenone) Package Label, 2010.

[40] Duramed, Mircette (ethinyl estradiol/desogestrel) Package Label, 2009.

[41] GlaxoSmithKline, Agenerase (amprenavir) Package Label, 2002.

[42] Abbott Laboratories, Norvir (ritonavir) Package Label, 2010.

[43] Janssen Therapeuics, Prezista (darunavir) Package Label, 2008.

[44] Boehringer Ingelheim Pharmaceuticals, Aptivus (tipranavir) Package Label, 2011.

[45] Roche Laboratories, Invirase/Fortovase (saquinavir) Package Label, 2010.

[46] M. Fröhlich, J. Burhenne, M. Martin-Facklam et al., "Oral contraception does not alter single dose saquinavir pharmacokinetics in women," British Journal of Clinical Pharmacology, vol. 57, no. 3, pp. 244-252, 2004.

[47] S. Abel, D. Russell, L. A. Whitlock, C. E. Ridgway, and G. J. Muirhead, "Effect of maraviroc on the pharmacokinetics of midazolam, lamivudine/zidovudine, and ethinyloestradiol/levonorgestrel in healthy volunteers," British Journal of Clinical Pharmacology, vol. 65, no. 1, pp. 19-26, 2008.

[48] Merck and Company, Isentress (raltegravir) Package Label, 2011.

[49] Organon, NuvaRing Package Label, 2010.

[50] Janssen-Ortho, Micronor (norethindrone) Package Label, 2010.

[51] Pfizer, Depo-Provera (depot medroxyprogesterone acetate) Package Label, 2006.

[52] D. H. Watts, J. G. Park, S. E. Cohn et al., "Safety and tolerability of depot medroxyprogesterone acetate among HIV-infected women on antiretroviral therapy: ACTG A5093," Contraception, vol. 77, no. 2, pp. 84-90, 2008.

[53] Bayer HealthCare Pharmaceuticals, Mirena (levonorgestrelreleasing intrauterine system) Package Label, 2009.

[54] Barr Pharmaceuticals, Plan B (levonorgestrel emergency contraceptive tablet) Product Labeling, 2006.

[55] J. Trussell and E. G. Raymond, "Emergency Contraception: a last chance to prevent unintended pregnancy," 2012, http://ec.princeton.edu/questions/ec-review.pdf.

[56] F. H. Stewart, J. Trussell, and P. F. A. Van Look, "Chapter 6: emergency contraception," in Contraceptive Technology, R. A. Hatcher, J. Trussell, A. L. Nelson, W. Cates Jr., F. H. Stewart, and D. Kowal, Eds., pp. 87-116, Ardent Media, New York, NY, USA, 19th edition, 2008.

[57] T. Juday, S. Gupta, K. Grimm, S. Wagner, and E. Kim, "Factors associated with complete adherence to HIV combination antiretroviral therapy," HIV Clinical Trials, vol. 12, no. 2, pp. 71-78, 2011. 
[58] M. J. Atkinson and J. J. Petrozzino, "An evidence-based review of treatment-related determinants of patients' nonadherence to HIV medications," AIDS Patient Care and STDs, vol. 23, no. 11, pp. 903-914, 2009.

[59] World Health Organization, Department of Reproductive Health and Research, "Hormonal contraception and HIV," Technical statement, Geneva, Switzerland, 2012.

[60] R. Heffron, D. Donnell, H. Rees et al., "Use of hormonal contraceptives and risk of HIV-1 transmission: a prospective cohort study," The Lancet Infectious Diseases, vol. 12, no. 1, pp. 19-26, 2012.

[61] World Health Organization, Medical Eligibility Criteria for Contraceptive Use, World Health Organization, Geneva, Switzerland, 2010. 


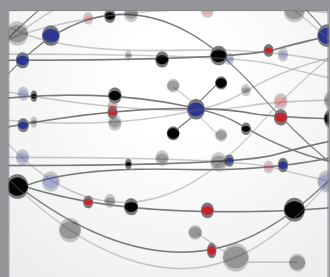

The Scientific World Journal
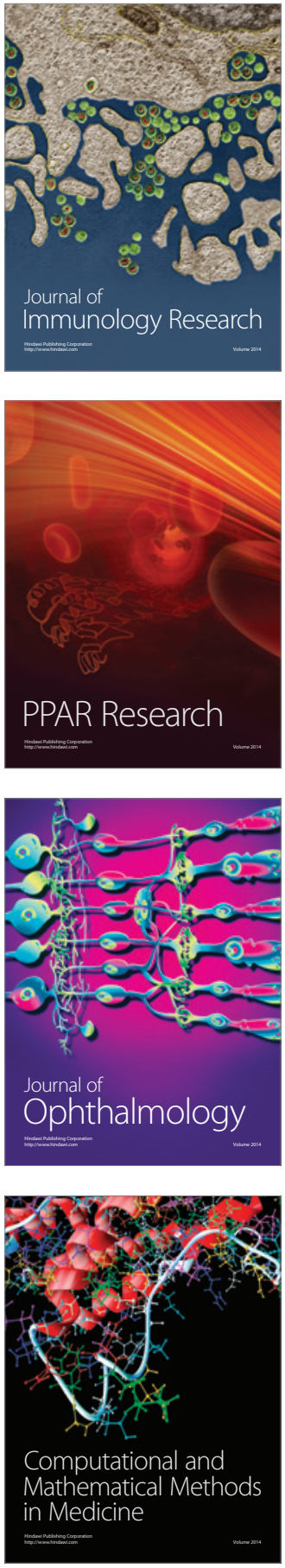

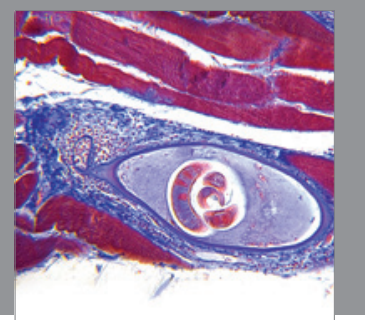

Gastroenterology

Research and Practice
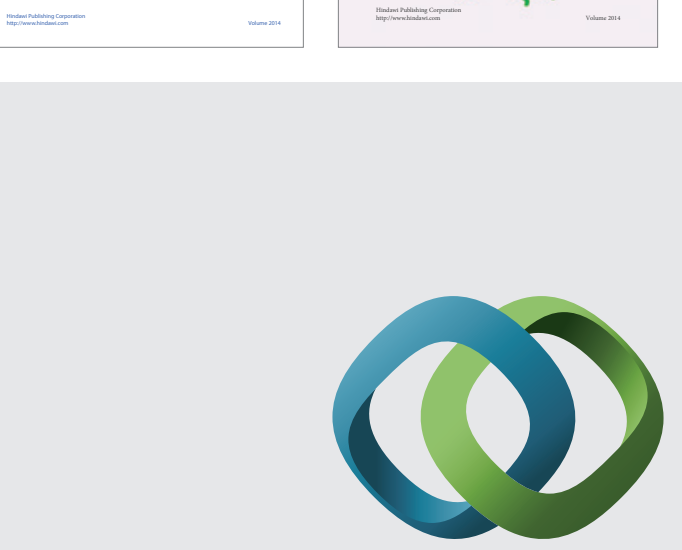

\section{Hindawi}

Submit your manuscripts at

http://www.hindawi.com
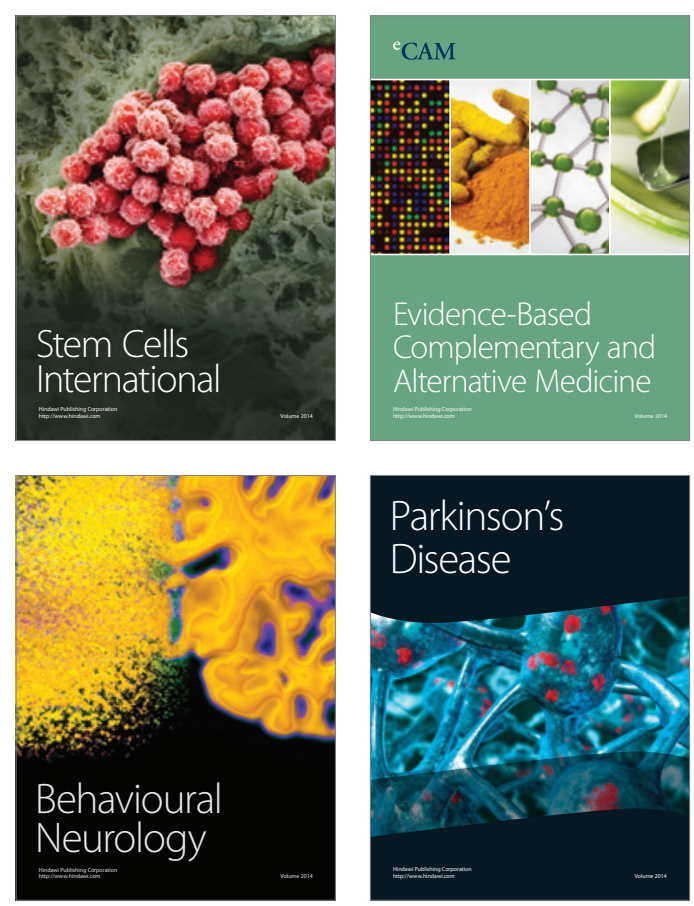

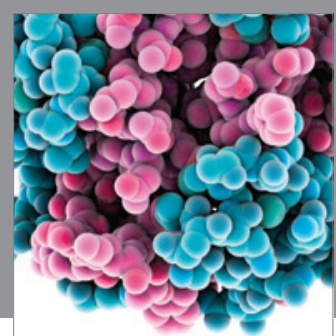

Journal of
Diabetes Research

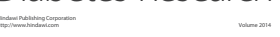

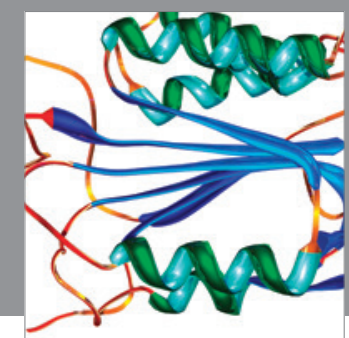

Disease Markers
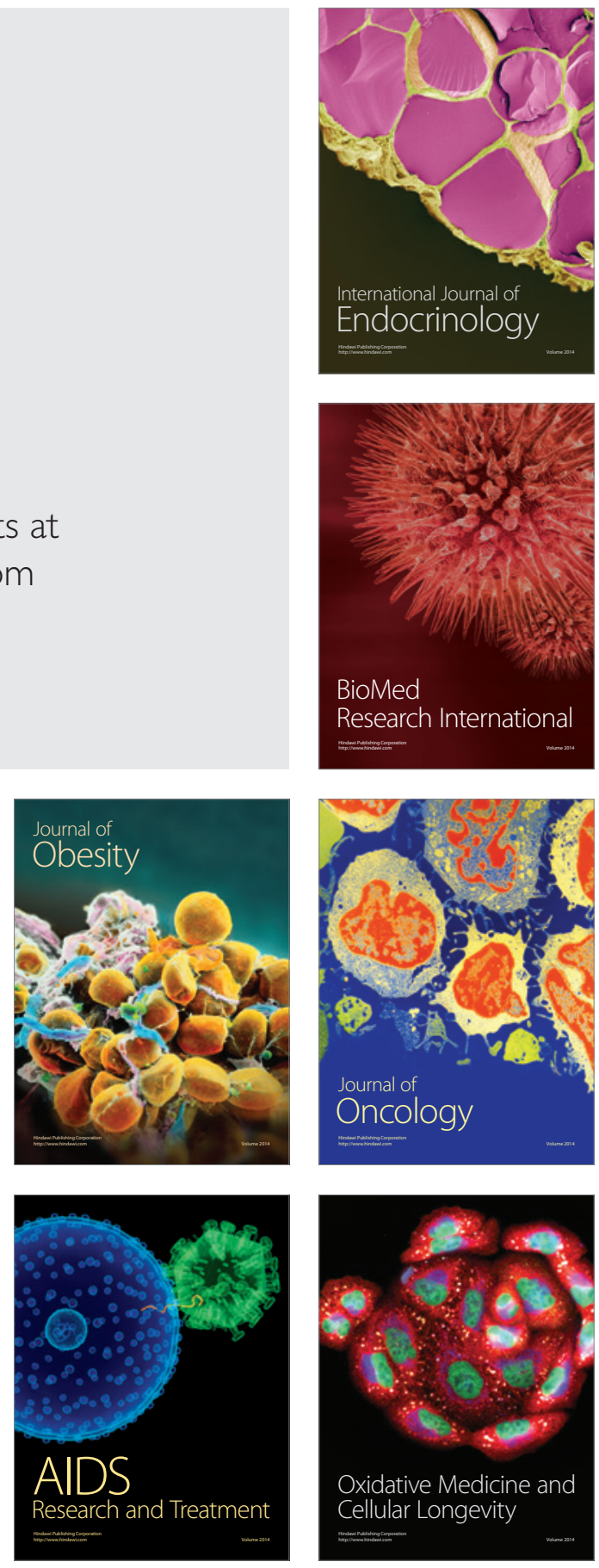